\title{
Measurement of Charm and Beauty Jets in Deep Inelastic Scattering at HERA
}

\section{P. Thompson*}

University of Birmingham

E-mail: pdt@hep.ph.bham.ac.uk

\section{On behalf of the $\mathrm{H} 1$ Collaboration}

\begin{abstract}
Measurements of cross sections for events with charm and beauty jets in deep inelastic scattering at HERA are presented. Events with jets of transverse energy $E_{T}^{\text {jet }}>6 \mathrm{GeV}$ and pseudorapidity $-1.0<\eta^{\text {jet }}<1.5$ in the laboratory frame are selected in the kinematic region of photon virtuality $Q^{2}>6 \mathrm{GeV}^{2}$ and inelasticity variable $0.07<y<0.625$. Measurements are also made requiring a jet in the Breit frame with $E_{T}^{* j e t}>6 \mathrm{GeV}$. The data were collected with the $\mathrm{H} 1$ detector in the years 2006 and 2007 corresponding to an integrated luminosity of $189 \mathrm{pb}^{-1}$. The numbers of charm and beauty jets are determined using variables reconstructed using the H1 vertex detector with which the impact parameters of the tracks to the primary vertex and the position of secondary vertices are measured. The measurements are compared with QCD predictions and with previous measurements where heavy flavours are identified using muons
\end{abstract}

XVIII International Workshop on Deep-Inelastic Scattering and Related Subjects, DIS 2010 April 19-23, 2010

Firenze, Italy

\footnotetext{
* Speaker.
} 


\section{Introduction}

The production of heavy flavour quarks in deep inelastic scattering (DIS) at the HERA electronproton collider is of particular interest for testing calculations in the framework of perturbative quantum chromodynamics (QCD). The process has the special feature of involving two hard scales: the square root of the photon virtuality $Q$ and the heavy quark mass $m$. In the case of jet production the transverse energy $E_{T}$ of the jet provides a further hard scale. In leading order (LO) QCD, the photon-gluon fusion (PGF) processes $e p \rightarrow e c \bar{c} X$ and $e p \rightarrow e b \bar{b} X$ are the dominant production mechanisms for charm $(c)$ and beauty $(b)$ quarks respectively.

The preliminary analysis presented here [1] reports on measurements by the $\mathrm{H} 1$ collaboration of the cross sections for events with a $c$ or $b$ jet in DIS at HERA. The analysis uses an inclusive lifetime technique following a similar procedure as used in [2] to distinguish the jets that contain $c$ and $b$ flavoured hadrons from those containing light flavoured hadrons.

\section{QCD models}

The jet cross section data are compared here with the predictions of the NLO QCD program HVQDIS [3]. The program is based on the PGF $\mathscr{O}\left(\alpha_{s}^{2}\right)$ matrix element and provides weighted events with two or three outgoing partons, i.e. a heavy quark pair and possibly an additional light parton. The calculations are made using quark masses: $m_{c}=1.5 \mathrm{GeV}, m_{b}=4.75 \mathrm{GeV}$. At NLO the predictions of QCD depend on the choice of the scales $\mu_{r}$ and $\mu_{f}$. To investigate the dependence of the predictions on the scales two example choices are made. Firstly, the scale $\mu_{r}=\mu_{f}=\sqrt{\left(Q^{2}+p_{T}^{2}+m^{2}\right) / 2}$, where $p_{T}$ is the transverse momentum of the heavy quark with the highest value of $p_{T}$ in the virtual photon-parton centre of mass frame, is used. Secondly, the scale $\mu_{r}=\mu_{f}=\sqrt{Q^{2}+4 m^{2}}$ is selected. Since HVQDIS provides cross sections at the parton level, corrections to the hadron level are needed in order to compare to the data. These corrections are calculated using the RAPGAP [ [ Monte Carlo event generator. In each kinematic bin of the measurement, the ratio $C_{\text {had }}$ of the RAPGAP hadron level to parton level cross sections is calculated and applied as a correction factor to the NLO calculation. The hadron level corrections generally amount to a change in the prediction by $\leq 6 \%$ for charm and $\leq 15 \%$ for beauty.

\section{Experimental Method}

Jets are reconstructed using the inclusive longitudinally invariant $k_{T}$ algorithm with the massless $P_{T}$ recombination scheme and the distance parameter $R_{0}=1$ in the $\eta-\phi$ plane. The algorithm is run in the laboratory frame using all reconstructed HFS particles and the resultant jets are required to have transverse energy $E_{T}^{\text {jet }}>1.5 \mathrm{GeV}$, in the angular range $-1.0<\eta^{\text {jet }}<1.5$. The $\eta$ range is asymmetric since the $y$ range chosen means few jets have $\eta<-1.0$.

The Monte Carlo simulation is also used to define hadron and parton level jets before they are processed by the simulation of the detector response. Hadron level jets are defined by running the same jet algorithm as for reconstructed jets using all final state particles, including neutrinos, but excluding the scattered electron. A Monte Carlo jet at the reconstructed or hadron level is defined as a ' $b$ jet' if there is at least one $b$ hadron within a cone of radius 1 about the jet axis in the $\eta-\phi$ 

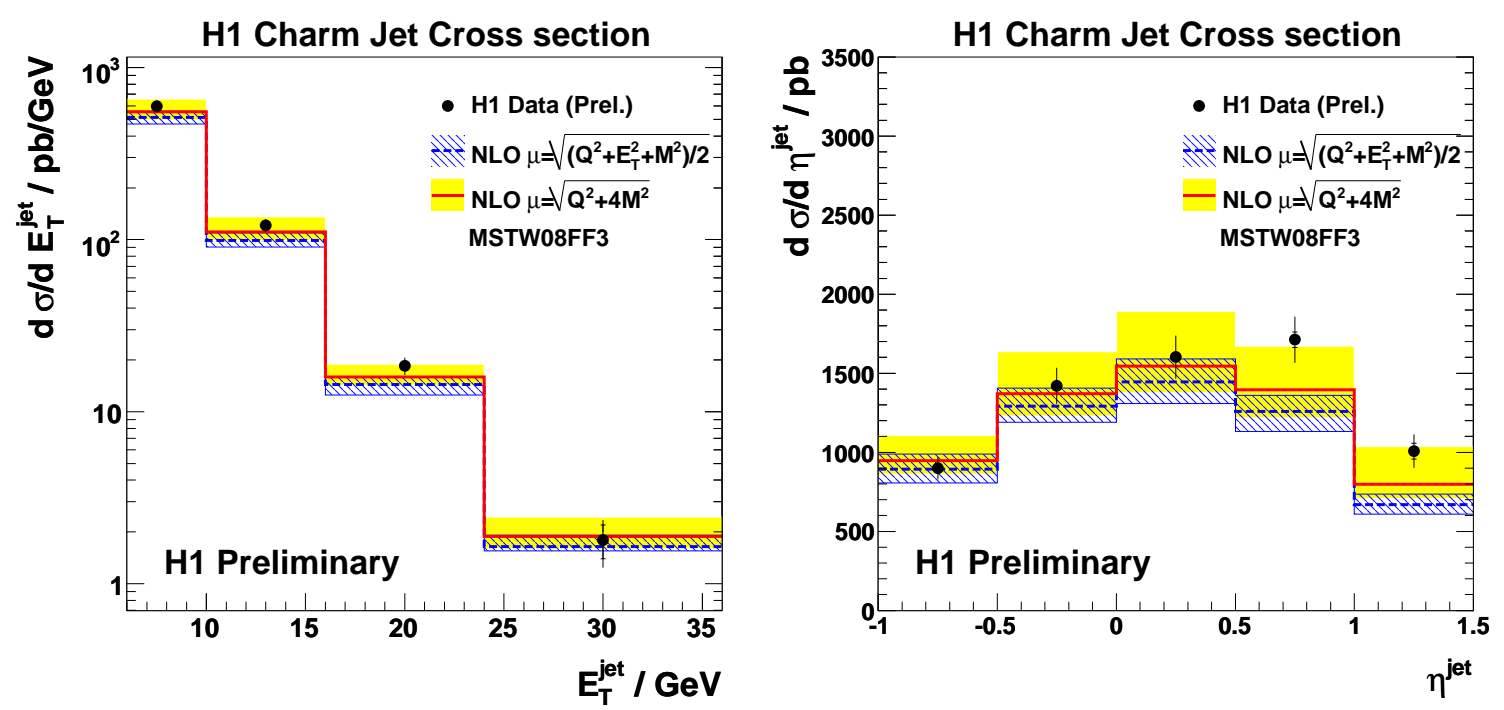

Figure 1: The cross sections for $c$ jet production in the laboratory frame as a function of $E_{T}^{\text {jet }}$ and $\eta^{\text {jet }}$. The data are compared with the predictions of NLO QCD.

plane. A jet is defined as a ' $c$ jet' if there is at least one $c$ hadron within the same cone and that $c$ hadron does not arise from the decay of a $b$ hadron. Jets that have not been classified as $c$ or $b$ jets are called 'light jets'. Parton level jets are defined for the Monte Carlo samples and for the NLO calculation by running the same jet algorithm on final state partons. A parton level jet is defined as a $b$ jet if there is at least one $b$ quark within a cone of radius 1 about the jet axis in the $\eta-\phi$ plane. A parton level jet is defined as a $c$ jet if there is at least one $c$ quark and no $b$ quark within the cone.

The separation of $b, c$ and light jets using the $\mathrm{H} 1$ vertex detector follows closely the procedure fully described in [2]. The separation is performed using the properties of well measured silicon tracks which are within a cone of radius 1 from the jet axis in the $\eta-\phi$ plane. The number of these tracks in the jet is called $N_{\text {track }}$. The impact parameter $\delta$ of a track is the transverse distance of closest approach of the track to the position of the beam interaction region. The sign of $\delta$ for a track is defined with respect to the azimuthal angle of the jet [2]. The track significance $S$ is defined as $S=\delta / \sigma(\delta)$, where $\sigma(\delta)$ is the uncertainty on $\delta$. The significances $S_{1}, S_{2}$ and $S_{3}$ are defined as the significance of the track with the highest, second highest and third highest absolute significance, respectively.

The $S_{1}$ distribution is used for jets where $N_{\text {track }}=1$ or $S_{1}$ and $S_{2}$ have opposite signs. The $S_{2}$ distribution is used for the remaining jets with $N_{\text {track }}=2$ or where $S_{3}$ has a different sign to $S_{1}$ and $S_{2}$. For jets with $N_{\text {track }} \geq 3$ where $S_{1}, S_{2}$ and $S_{3}$ all have the same sign an artificial neural network (NN) is used to produce a distribution that combines several variables in order to provide an optimal discrimination between $b$ and $c$ jets. The inputs to the $\mathrm{NN}$ are $S_{1}, S_{2}, S_{3}$, the significance of the transverse distance between the secondary and primary vertex, the transverse momenta of the tracks with the highest and second highest transverse momentum, the number of selected silicon tracks and the number of reconstructed tracks at the secondary vertex. The fractions of events with $c, b$ and light jets in the data are extracted using a least squares simultaneous fit to the $S_{1}, S_{2}$ and NN output distributions, and the total number of events after DIS and jet selection. 

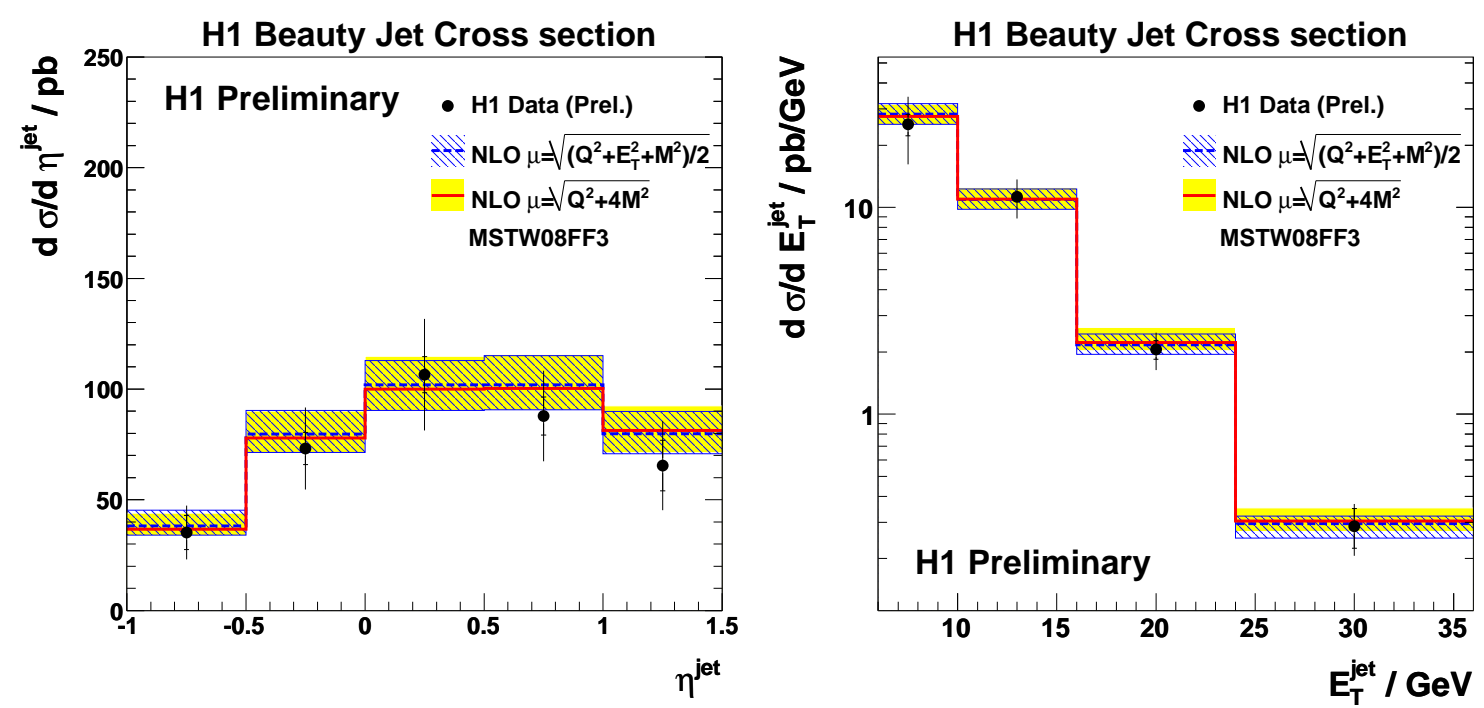

Figure 2: The cross sections for $b$ jet production in the laboratory frame as a function of $E_{T}^{\text {jet }}$ and $\eta^{\text {jet }}$. The data are compared with the predictions of NLO QCD.

\section{Results}

The cross sections for $c$ and $b$ jets in the laboratory frame are measured in the kinematic range $Q^{2}>6 \mathrm{GeV}^{2}$ and $0.07<y<0.625$ for the heavy flavour jet with the highest $E_{T}^{\mathrm{jet}}$ with $E_{T}^{\mathrm{jet}}>6 \mathrm{GeV}$ and $-1.0<\eta^{\text {jet }}<1.5$. The differential $c$ jet cross sections are shown as a function of $E_{T}^{\text {jet }}$ and $\eta^{\text {jet }}$ in figure 1. The data are also measured as a function of $Q^{2}$ and the number of jets $N_{\text {jet }}$. The data are compared with the NLO predictions of HVQDIS including hadronisation corrections. In general the NLO expectations describe the charm data reasonably well in all differential distributions although the predictions with the scale $\mu=\sqrt{\left(Q^{2}+p_{T}^{2}+m^{2}\right) / 2}$ fall somewhat below the data at low $E_{T}^{\text {jet }}$ and in the forward $\eta^{\text {jet }}$ region. The differential $b$ jet cross sections in the laboratory frame are shown as a function of $E_{T}^{\text {jet }}$ and $\eta^{\text {jet }}$ in figure 2. HVQDIS gives a good description of the beauty data with little dependence on the choice of scale.

The data were also compared with the predictions of Monte Carlo programs based on leading order matrix elements with the effect of higher orders modelled by initial and final state parton showers. The predictions of the RAPGAP Monte Carlo program are found to give a good description of the data. The Monte Carlo CASCADE [5], based on the CCFM evolution equation, is found to overshoot the charm data at small $Q^{2}$, and high $\eta^{\text {jet }}$. It provides a better prediction of the beauty cross section normalisation than it does for charm but still tends to overestimate the data at low $Q^{2}$ and high $\eta^{\text {jet }}$.

The $b$ jet cross sections, measured in the Breit frame, are also compared with $b$ jet measurements obtained from muon tagging by H1 [6] and ZEUS [7]. The Breit frame $b$ jet cross sections are compared as a function of $Q^{2}$ in figure 3. The muon data are corrected for the $b \rightarrow \mu$ branching fraction and for the extrapolation from the phase space of the muon measurement, which had restrictions on $p_{T}^{\mu}$ and $\eta^{\mu}$, to the phase space of the present analysis. An additional uncertainty of around $10 \%$ is added to the corrected muon measurements to account for theoretical uncertainties 
H1 Beauty Breit Frame Jet Cross section

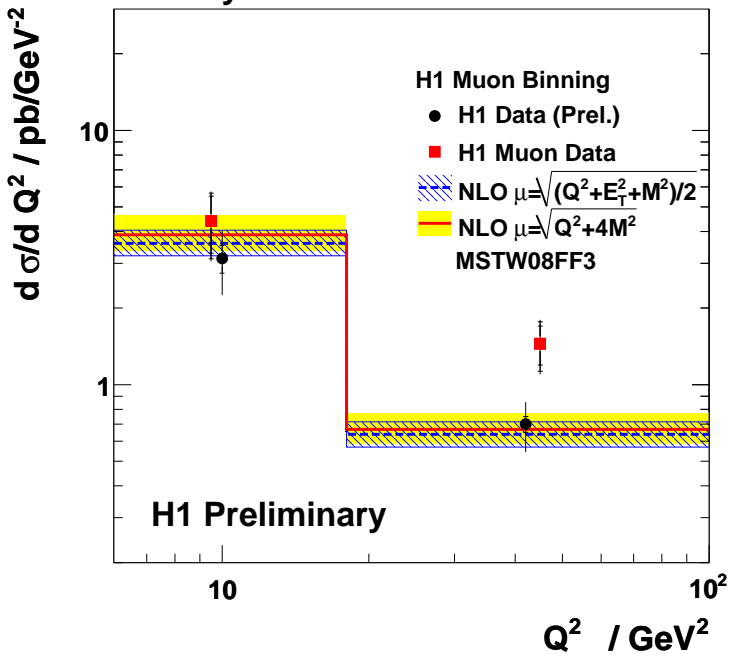

H1 Beauty Breit Frame Jet Cross section

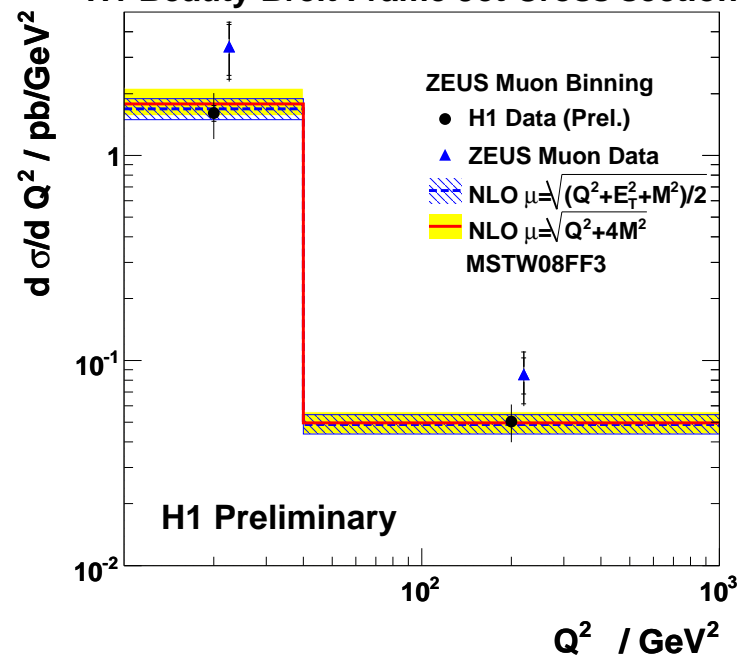

Figure 3: The cross sections for $b$ jet production in the Breit frame as a function of $Q^{2}$. The data are compared with data extrapolated from measurements obtained using muon tagging from H1 [6] (left) and ZEUS 沛] (right). The predictions of NLO QCD are also shown.

on the extrapolation factors coming from uncertainties on the perturbative scales and fragmentation model used. The central values of the present data in the Breit frame tend to lie below the adjusted H1 muon data at high $Q^{2}$ and below the ZEUS muon data at low $Q^{2}$.

\section{Summary}

The present heavy flavour jet measurements show that charm and beauty production in deep inelastic scattering is adequately described by NLO QCD in the presence of an additional hard scale provided by a jet.

\section{References}

[1] [H1 Collaboration], H1 Preliminary H1prelim-10-073.

[2] F. D. Aaron et al. [H1 Collaboration], Eur. Phys. J. C 65 (2010) 89 [arXiv:0907.2643];

A. Aktas et al. [H1 Collaboration], Eur. Phys. J. C 40 (2005) 349 [hep-ex/0411046].

[3] B. W. Harris and J. Smith, Phys. Rev. D 57 (1998) 2806 [hep-ph/9706334];

B. W. Harris and J. Smith, Nucl. Phys. B 452 (1995) 109;

B. W. Harris and J. Smith, Phys. Lett. B 353 (1995) 535.

[4] RAPGAP 3.1, H. Jung, Comput. Phys. Commun. 86 (1995) 147; (see also http://www.desy.de/ jung/rapgap/).

[5] H. Jung and G. P. Salam, Eur. Phys. J. C 19 (2001) 351 [hep-ph/0012143].

[6] A. Aktas et al. [H1 Collaboration], Eur. Phys. J. C 41 (2005) 453 [hep-ex/0502010].

[7] S. Chekanov et al. [ZEUS Collaboration], Phys. Lett. B 599 (2004) 173 [hep-ex/0405069]. 